\title{
Predicting the chances of a live birth after one or more complete cycles of in vitro fertilisation: population based study of linked cycle data from 113873 women
}

\author{
David J McLernon,, Ewout W Steyerberg, ${ }^{2}$ Egbert R te Velde, ${ }^{2}$ Amanda J Lee, ${ }^{1}$ Siladitya Bhattacharya ${ }^{3}$
}

$\overline{{ }^{1} \text { Medical Statistics Team, Institute }}$ of Applied Health Sciences, University of Aberdeen, Aberdeen AB25 2ZD, UK

2Department of Public Health, Erasmus MC-University Medical Centre Rotterdam, Rotterdam, Netherlands

${ }^{3}$ Institute of Applied Health Sciences, University of Aberdeen, Aberdeen, UK Correspondence to: D J McLernon d.mclernon@abdn.ac.uk Additional material is published online only. To view please visit the journal online.

Cite this as: BMJ 2016;355:i5735 http://dx.doi.org/10.1136/bmj.i5735

Accepted: 19 October 2016

\section{ABSTRACT}

\section{OBJECTIVE}

To develop a prediction model to estimate the chances of a live birth over multiple complete cycles of in vitro fertilisation (IVF) based on a couple's specific characteristics and treatment information.

DESIGN

Population based cohort study.

SETTING

All licensed IVF clinics in the UK. National data from the Human Fertilisation and Embryology Authority register. PARTICIPANTS

All 253417 women who started IVF (including intracytoplasmic sperm injection) treatment in the UK from 1999 to 2008 using their own eggs and partner's sperm.

\section{MAIN OUTCOME MEASURE}

Two clinical prediction models were developed to estimate the individualised cumulative chance of a first live birth over a maximum of six complete cycles of IVF-one model using information available before starting treatment and the other based on additional information collected during the first IVF attempt. A complete cycle is defined as all fresh and frozenthawed embryo transfers arising from one episode of ovarian stimulation.

RESULTS

After exclusions, 113873 women with 184269 complete cycles were included, of whom 33154 (29.1\%) had a live birth after their first complete cycle and 48925

\section{WHAT IS ALREADY KNOWN ON THIS TOPIC}

Although the success of in vitro fertilisation (IVF) has generally been reported as the livebirth rate in a single fresh embryo transfer, the increase in embryo cryopreservation has meant that cumulative livebirth rates are more informative Previous prediction models have either only estimated the individualised chances of a live birth after a single fresh embryo transfer or ignored the contribution of frozen embryo transfers to the cumulative chances of a live birth

Failure to take account of all embryo transfer attempts has meant that such prediction models have not proved to be useful as counselling tools as the first treatment does not always succeed

\section{WHAT THIS STUDY ADDS}

Our novel prediction models estimate the cumulative chances of a live birth over six complete cycles (including replacement of fresh and frozen embryos) of IVF before treatment and after the first fresh embryo transfer attempt

The cumulative aspect of our prediction models will aid clinicians by supporting their clinical knowledge when communicating to couples their personalised chances of a live birth over an entire package of IVF treatment.

The cumulative aspect of our prediction models will also help couples plan their time and prepare emotionally and financially for their complete IVF journey
(43.0\%) after six complete cycles. Key pretreatment predictors of live birth were the woman's age (31 v 37 years; adjusted odds ratio $1.66,95 \%$ confidence interval 1.62 to 1.71$)$ and duration of infertility ( $3 v 6$ years; $1.09,1.08$ to 1.10$)$. Post-treatment predictors included number of eggs collected (13 $\vee 5$ eggs; 1.29 , 1.27 to 1.32$)$, cryopreservation of embryos $(1.91,1.86$ to 1.96), the woman's age $(1.53,1.49$ to 1.58$)$, and stage of embryos transferred (eg, double blastocyst $v$ double cleavage; $1.79,1.67$ to 1.91). Pretreatment, a 30 year old woman with two years of unexplained primary infertility has a $46 \%$ chance of having a live birth from the first complete cycle of IVF and a $79 \%$ chance over three complete cycles. If she then has five eggs collected in her first complete cycle followed by a single cleavage stage embryo transfer (with no embryos left for freezing) her chances change to $28 \%$ and $56 \%$, respectively.

\section{CONCLUSIONS}

This study provides an individualised estimate of a couple's cumulative chances of having a baby over a complete package of IVF both before treatment and after the first fresh embryo transfer. This novel resource may help couples plan their treatment and prepare emotionally and financially for their IVF journey.

\section{Introduction}

In the United Kingdom, one in six couples experience difficulties conceiving. ${ }^{1}$ In vitro fertilisation (IVF) is the recommended treatment of choice for all couples with continued unresolved fertility problems. ${ }^{2}$ By the end of 2013 over five million people were born as a result of IVF or intracytoplasmic sperm injection (ICSI). ${ }^{3}$

Although the success of IVF and ICSI has conventionally been reported as the livebirth rate in a single fresh cycle, ${ }^{45}$ widespread use of embryo cryopreservation in the past two decades has meant that cumulative livebirth rates, which include frozen embryo replacements as well as subsequent treatment episodes, are more informative, as they empower couples and clinicians to make strategic decisions about care over a period of time. ${ }^{6}$ However, cumulative livebirth rates are often reported either as one overall average rate or stratified by the woman's age or type of infertility at a national or IVF clinic level. ${ }^{7-11}$ Given that many other patient and treatment characteristics can affect the success of IVF or ICSI, ${ }^{12}$ it can be difficult for clinicians to counsel couples as to their individualised chances of success over a complete package of IVF or ICSI treatment.

A clinical prediction model that could estimate the cumulative chances of a live birth before IVF or ICSI begins and that could provide a revised estimate of the 
expected outcomes after one or more treatment cycles would be critical for patient counselling and informing decision making in IVF. No such model currently exists. We therefore estimated the predicted cumulative probability of a first live birth over multiple complete cycles of IVF or ICSI at initial consultation before treatment starts (pretreatment) and after the first attempt at fresh embryo transfer, when treatment specific information is available (post-treatment).

\section{Methods}

Database

The Human Fertilisation and Embryology Authority (HFEA) has collected data on all licensed fertility treatments in the UK since 1992. An anonymised HFEA database is freely available online for research purposes. ${ }^{13-16}$ This version only contains data at the individual (fresh or frozen) IVF treatment level, with no link between treatment and individual women-a requirement when estimating the cumulative probability of a live birth. However, a more detailed version of the HFEA database is available for research purposes under strict conditions, which links all IVF treatments to complete cycles and to individual women and allows estimation of the cumulative probability of a live birth. ${ }^{15} 17$ Following approval by the North of Scotland research ethics committee, the Confidentiality Advisory Group, and the HFEA register research panel, anonymised data on all treatments linked to each woman undergoing IVF or ICSI were made available to the authors.

\section{Study population}

In this population based cohort study, records of all complete IVF and ICSI cycles-defined as all attempts at fresh and frozen embryo transfer resulting from one episode of ovarian stimulation-in women who started their first ovarian stimulation in the UK between January 1999 and September 2008 were extracted up until September 2009 when exposure to IVF ended. Data from October 2009 onwards were not included in the analysis because consent for patient data on IVF or ICSI to be used in research changed from "presumed" to "required" at that point. Including such years would have led to falsely higher discontinuation rates owing to women opting not to disclose their treatment information in later complete cycles. Women who had any element of treatment involving donor insemination, egg donation, and surrogacy were not included in the requested database. Supplementary figure A1 details further exclusion criteria made on receipt of the data.

\section{Baseline characteristics}

Baseline characteristics of couples included the woman's age, duration of infertility (years), type of infertility (categorised as diagnosis of tubal, endometriosis, anovulation, male factor, or unexplained), previous pregnancy status of the couple (yes versus no), treatment type (ICSI versus IVF), and year of first egg retrieval. Treatment level characteristics from the attempt at fresh embryo transfer of the first complete cycle included number of eggs collected, number of embryos transferred, stage of embryo transfer (cleavage versus blastocyst), and whether embryos were frozen (cryopreservation of embryos).

\section{Outcome}

We estimated the cumulative chances of a first live birth for a couple having up to six complete cycles of IVF. One complete cycle included all fresh and frozen embryo transfers resulting from one episode of ovarian stimulation.

\section{Statistical analysis}

We developed two clinical prediction models to use before and after treatment.

Pretreatment model-for a couple about to embark on IVF or ICSI, we estimated the cumulative probability of a live birth over a maximum of six complete cycles using the characteristics of the couple and whether they planned to undergo IVF or ICSI.

Post-treatment model-to update the cumulative probability of live birth after the first attempt at embryo transfer, we included treatment specific characteristics from the first complete cycle along with the characteristics of the couple from the pretreatment model.

\section{Model development}

We used a discrete time logistic regression model to predict the chance of a live birth after a maximum of six cumulative complete cycles of IVF or ICSI, where a complete cycle included a fresh embryo transfer and any associated frozen-thawed embryo transfers. To assess the predicted probability of a live birth in the ith complete cycle conditional on no live birth having occurred before that complete cycle, we treated the complete cycle number as a discrete time variable. From this model, we calculated the cumulative probability of a live birth over sequential complete cycles up to complete cycle 6 .

Initially we fitted univariable models to assess the effect of individual predictors (adjusting for complete cycle number) on live birth. The woman's age, treatment year, and number of eggs retrieved all had nonlinear relations with the probability of a live birth so were modelled using restricted cubic splines. Effect estimates were presented for the interquartile range (25th $v$ 75th centile values, or 75 th $v 25$ th centile values). ${ }^{18}$

A manual backward selection process was used to determine the final multivariable discrete time logistic regression model to predict live birth over successive complete cycles of IVF or ICSI. In this procedure, complete cycle number, the woman's age, duration of infertility, and number of eggs collected (for post-treatment model only) were always included and could not be removed because these are known predictors of pregnancy outcomes after IVF. ${ }^{12}$ All of the other available predictors (listed as baseline characteristic) were subjected to the selection process. Women who had no eggs collected were excluded from the post-treatment model because it would not be possible to achieve a live birth in the first complete cycle. Therefore we fitted a separate model for these women. Since IVF centres have varying success rates, we also considered a random intercept for 
the effect of IVF centre as recommended in recent studies. ${ }^{1920}$ To quantify variation between centres, we calculated the $95 \%$ range for centre effect.

\section{Missing data}

Where data were missing for any predictor, we compared the characteristics of women with complete data with those for women with missing data. Single imputation was performed for those predictors with missing information. This procedure assumes that missingness was random, conditional on the observed predictors and outcome.

\section{Predictive ability}

To determine the explanatory value of each predictor relative to the entire set we calculated the adequacy

\begin{tabular}{|c|c|}
\hline Characteristics & Data \\
\hline No of women & 113873 \\
\hline No of complete cycles & 184269 \\
\hline \multicolumn{2}{|l|}{ Patient characteristics } \\
\hline Mean (SD) age of women & $34.1(5)$ \\
\hline Median (interquartile range) duration of infertility (years) & $4(3-6)$ \\
\hline Missing & $18225(16)$ \\
\hline No previous pregnancy in couple & $75541(66)$ \\
\hline Previous pregnancy in couple & $28070(25)$ \\
\hline Missing* & $10262(9)$ \\
\hline \multicolumn{2}{|l|}{ Type of infertility: } \\
\hline Tubal & $26545(23)$ \\
\hline Anovulatory & $15942(14)$ \\
\hline Male factor & $49753(44)$ \\
\hline Unexplained & $32693(29)$ \\
\hline Endometriosis & $7590(7)$ \\
\hline$>1$ type & $13414(12)$ \\
\hline \multicolumn{2}{|l|}{ Year of first egg retrieval: } \\
\hline $1999-2001$ & $29451(26)$ \\
\hline $2002-04$ & $34472(30)$ \\
\hline $2005-08$ & $49950(44)$ \\
\hline \multicolumn{2}{|l|}{ Treatment characteristics at complete cycle 1} \\
\hline IVF & $67511(59)$ \\
\hline Intracytoplasmic sperm injection & $46362(41)$ \\
\hline Median No (interquartile range) of eggs collected & $8(5-13)$ \\
\hline Median No (interquartile range) of embryos created & $5(2-8)$ \\
\hline Median No (interquartile range) of embryos frozen & $0(0-1)$ \\
\hline Cryopreservation of embryos & $28950(25)$ \\
\hline \multicolumn{2}{|l|}{ No of frozen treatments: } \\
\hline 0 & $103726(91)$ \\
\hline 1 & $8270(7)$ \\
\hline 2 & $1568(1)$ \\
\hline 3 & $246(0.2)$ \\
\hline$\geq 4$ & $63(0.1)$ \\
\hline \multicolumn{2}{|l|}{ No of embryos transferred and stage, first fresh treatment: } \\
\hline No transfer & $15501(14)$ \\
\hline Single cleavage stage & $9248(8)$ \\
\hline Single blastocyst stage & $662(1)$ \\
\hline Double cleavage stage & $75701(66)$ \\
\hline Double blastocyst stage & $2960(3)$ \\
\hline Triple cleavage stage & $8649(8)$ \\
\hline Triple blastocyst stage & $130(0.1)$ \\
\hline Missing & $1022(1)$ \\
\hline
\end{tabular}

statistic. ${ }^{21}$ The adequacy is the proportion of the total explained variation in the outcome that is explained by the individual predictor. ${ }^{22}$

We used the $\mathrm{C}$ index to assess the ability of the multivariable models to discriminate between couples at high and low chances of a live birth. ${ }^{23}$ Because the performance of the models with and without the random effect of centre were shown to be similar, we considered the fixed effects model to be the final model.

To assess whether the model calibration was too optimistic within the database from which the model was developed, we used bootstrapping for internal validation (see supplementary text S1). ${ }^{18}$ By internally validating the calibration slope of the model we could determine whether the model underestimated or overestimated predictions. ${ }^{18}$ A perfect slope should equal 1.

\section{Treatment continuation}

A further analysis was done to investigate the effect of predictors on continuing to a second complete cycle of IVF in women who did not have a live birth in complete cycle 1 . The methods and results of this are reported in the appendix (see supplementary text S2). Statistical analysis was carried out using SAS (V.9.3) (SAS Institute, Cary, NC).

\section{Patient involvement}

No patients were involved in setting the research question or the outcome measures, nor were they involved in developing plans for design or implementation of the study. No patients were asked to advise on interpretation or writing up of results. There are plans to disseminate the results of the research to patients affected by fertility issues via national fertility charities and the Human Fertilisation and Embryology Authority.

\section{Results}

After exclusions, the dataset included 113873 couples who underwent 184269 complete cycles of IVF or ICSI (see supplementary figure A1). Table 1 shows the distribution of the characteristics of the couples and those of their first complete cycle (defined as all fresh and frozen embryo transfers resulting from one episode of ovarian stimulation), which resulted in a live birth in 33154 (29\%) women (fig 1). Of the remaining 80719 couples only $45384(56 \%)$ went on to have a second complete cycle. Overall, $43 \%$ of couples had a live birth over the first six complete cycles of IVF or ICSI. The median time to follow-up for those not having a live birth was 1515 days (interquartile range 669-2631). Thirty per cent of all live births were multiples. Supplementary text S1 provides detailed information on missing data.

\section{Predicting the cumulative chances of a live birth} Individually, all patient and treatment level characteristics had statistically significant univariable associations with live birth, except for unexplained infertility (see supplementary table A1). Figure 2 shows the unadjusted relation between the predicted probability of live birth in the first complete cycle and the woman's age, duration of infertility, treatment year, and number of 


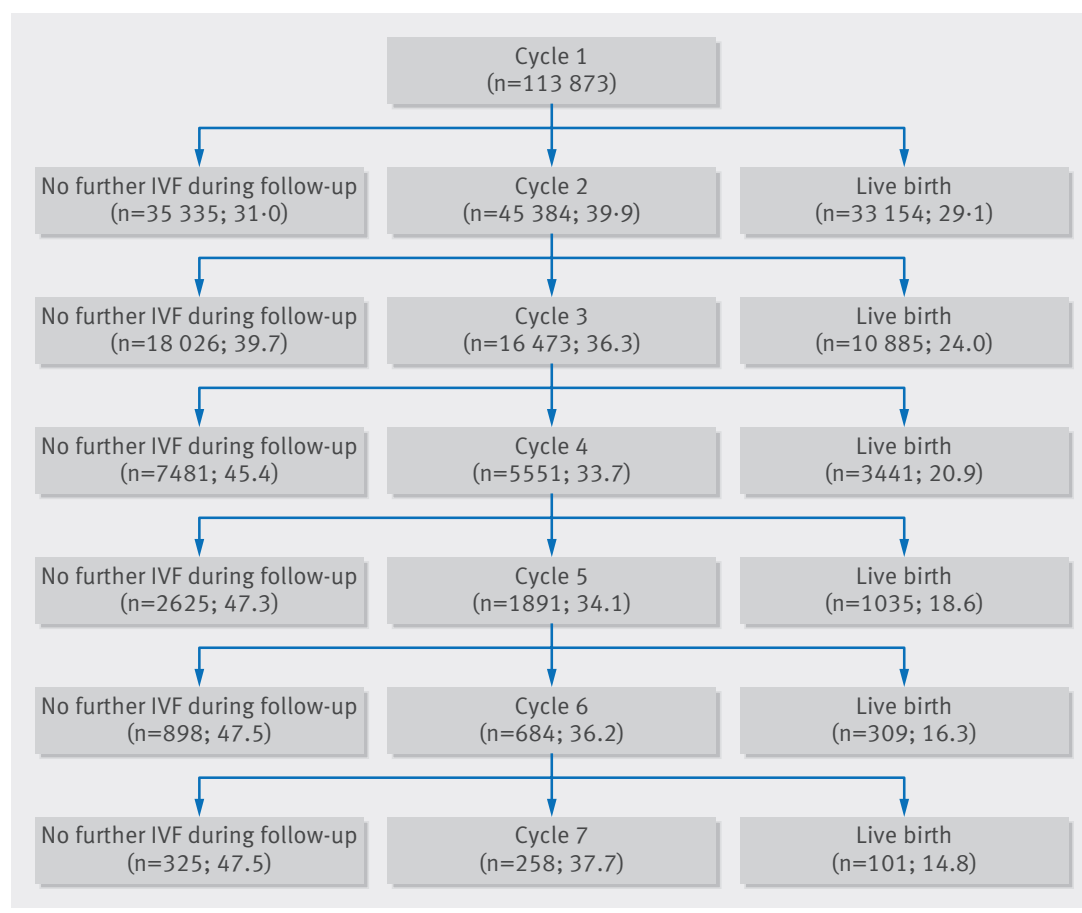

Fig 1 Number of women having a live birth, not having a live birth but continuing in vitro fertilisation (IVF), or not having a live birth and not continuing IVF (during follow-up) over six complete cycles of IVF eggs collected. The chances of a live birth declined after age 30 and decreased linearly with increasing duration of infertility. Increasing egg numbers improved the chance of live birth considerably until 13 eggs. Year of treatment showed a positive effect from 2006 onwards.

\section{Pretreatment model}

The factors that predicted the cumulative chances of having a live birth over a maximum of six complete cycles (in a multivariable model) were the woman's age, duration of infertility, treatment type, year, tubal infertility, male factor infertility, unexplained infertility, anovulation, and previous pregnancy, with age explaining $85 \%$ of the total variation explained by all the factors (table 2 and supplementary table A2). The odds of a live birth decreased with every increasing complete cycle-eg, the odds of a live birth after complete cycle 2 was $21 \%$ lower than the odds of a live birth after complete cycle 1 , and the odds after complete cycle 6 were $56 \%$ less than after complete cycle 1 . With increasing woman's age the odds of a live birth over six complete cycles decreased ( $31 v 37$ years; adjusted odds ratio 1.66, 95\% confidence interval 1.62 to 1.71 ). Increasing duration of infertility reduced the odds of a live birth ( $3 v 6$ years; $1.09,1.08$ to 1.10). Couples with a diagnosis of tubal or male factor infertility had around a 10\% reduced chance of a live birth, whereas couples with
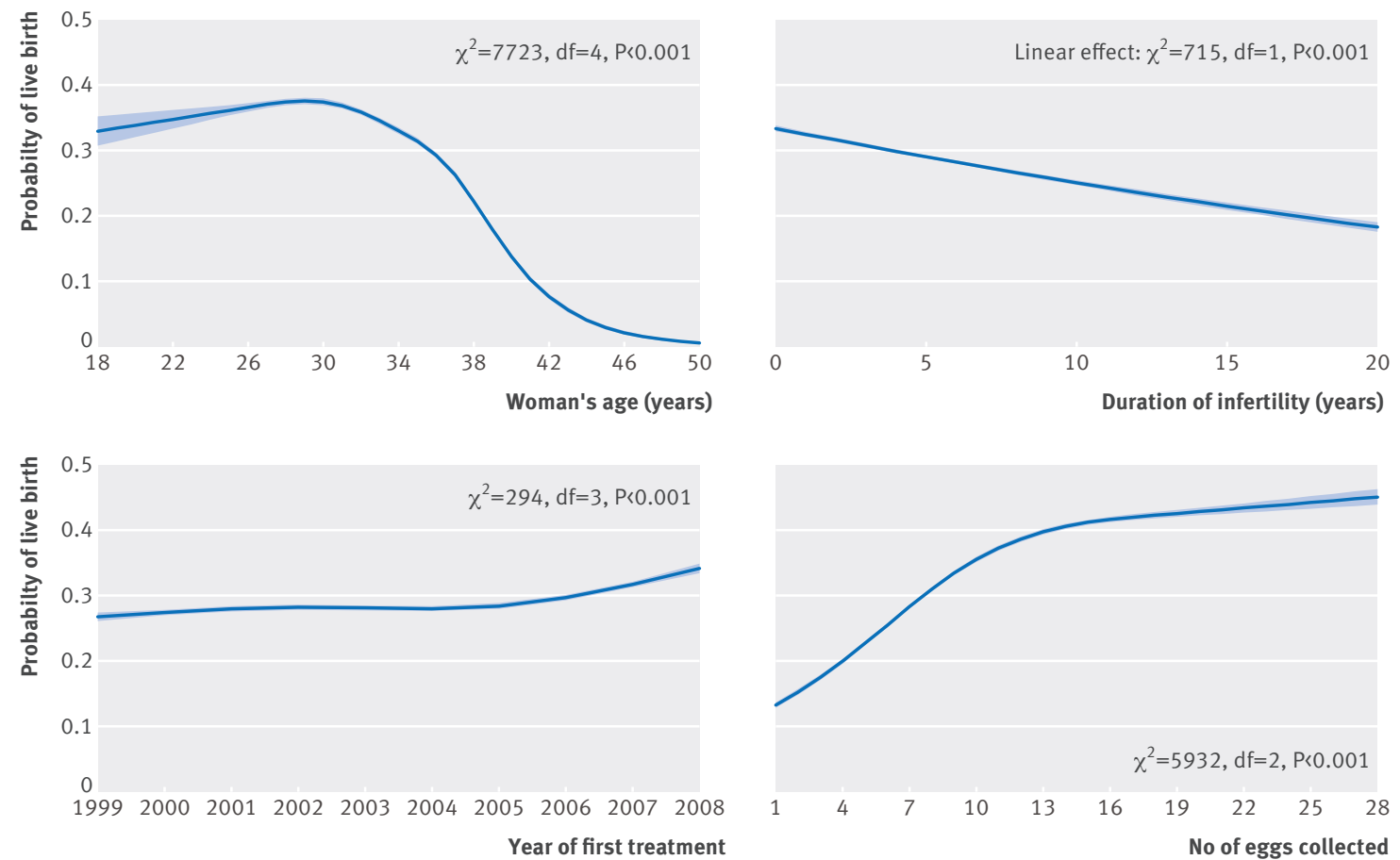

Fig 2 | Plots showing unadjusted (univariable) relations between the following continuous baseline variables and a live birth in the first complete cycle of in vitro fertilisation. (A) Woman's age (years); (B) duration of infertility (years); (C) year of first complete cycle, and (D) number of eggs retrieved in first complete cycle. Each panel depicts the probability of live birth (solid curve) with $95 \%$ confidence bands as a function of the baseline variable. The degrees of freedom (df) associated with the $\chi 2$ statistic in each case depend on the complexity of the relation between the variable and the log odds of live birth. Where $\mathrm{df}>1$, the relation was non-linear and characterised by restricted cubic spline functions. Where $\mathrm{df}=1$, the relation was linear even though the plot shown here, which reflects live birth (rather than the log odds), exhibits a non-linear relation 
Table 2 | Effect and importance (in decreasing order) of each predictor on live birth over multiple complete cycles of in vitro fertilisation (IVF) adjusted for patient characteristics (pretreatment model)

\begin{tabular}{|c|c|c|c|}
\hline Predictors & Odds ratio $(95 \% \mathrm{Cl})$ & P value & Adequac \\
\hline Intercept & & $<0.001$ & \\
\hline \multicolumn{4}{|l|}{ Complete cycle No: } \\
\hline 1 (reference) & 1 & & \\
\hline 2 & 0.79 (0.77 to 0.81$)$ & $<0.001$ & \\
\hline 3 & 0.66 (0.64 to 0.69$)$ & $<0.001$ & \\
\hline 4 & $0.57(0.53$ to 0.61$)$ & $<0.001$ & \\
\hline 5 & 0.49 (0.43 to 0.55$)$ & $<0.001$ & \\
\hline 6 & 0.44 (0.36 to 0.55$)$ & $<0.001$ & \\
\hline \multicolumn{4}{|l|}{ Patient characteristics: } \\
\hline Woman's age, 31 v 37 years* & $1.66(1.62$ to 1.71$)$ & $<0.001$ & 0.846 \\
\hline Duration, $3 v 6$ years & 1.09 (1.08 to 1.10$)$ & $<0.001$ & 0.078 \\
\hline Treatment type, ICSI v IVF & $1.24(1.21$ to 1.28$)$ & $<0.001$ & 0.047 \\
\hline Year first complete cycle started, 2006 v 2001* & $1.20(1.16$ to 1.24$)$ & $<0.001$ & 0.032 \\
\hline Tubal infertility, yes $v$ no & $0.91(0.88$ to 0.94$)$ & $<0.001$ & 0.012 \\
\hline Male factor infertility, yes $v$ no & $0.90(0.87$ to 0.94$)$ & $<0.001$ & 0.009 \\
\hline Unexplained infertility, yes $v$ no & $1.06(1.03$ to 1.10$)$ & $<0.001$ & 0.001 \\
\hline Anovulatory infertility, yes $v$ no & 1.05 (1.01 to 1.09$)$ & 0.01 & 0.001 \\
\hline Previous pregnancy in couple, no $v$ yes & 0.93 (0.90 to 0.95$)$ & $<0.001$ & 0.0002 \\
\hline \multicolumn{4}{|c|}{$\begin{array}{l}\text { ICSI=intracytoplasmic sperm injection. } \\
\text { *Interquartile odds ratio was calculated to aid interpretation of continuous predictors of age, duration of } \\
\text { infertility, and year first complete cycle started. It is defined as the ratio of the odds of a live birth for the } 75 \text { th } \\
\text { centile and the odds of a live birth for the } 25 \text { th centile of the predictor. }\end{array}$} \\
\hline
\end{tabular}

Table 3 | Effect and importance (in decreasing order) of each predictor on live birth over multiple complete cycles of in vitro fertilisation (IVF) adjusted for patient characteristics and treatment information at first complete cycle (post-treatment model)

\begin{tabular}{|c|c|c|c|}
\hline Predictors & Odds ratio $(95 \% \mathrm{Cl})$ & $P$ value & Adequacy \\
\hline Intercept & & $<0.001$ & \\
\hline \multicolumn{4}{|l|}{ Complete cycle No: } \\
\hline 1 (Reference) & 1 & & \\
\hline 2 & $0.82(0.80$ to 0.85$)$ & $<0.001$ & \\
\hline 3 & 0.70 (0.67 to 0.73$)$ & $<0.001$ & \\
\hline 4 & 0.60 (0.56 to 0.65$)$ & $<0.001$ & \\
\hline 5 & $0.51(0.44$ to 0.58$)$ & $<0.001$ & \\
\hline 6 & $0.46(0.37$ to 0.58$)$ & $<0.001$ & \\
\hline \multicolumn{4}{|l|}{ Patient characteristics } \\
\hline Woman's age to $31 v 37$ years* & 1.53 (1.49 to 1.58.$)$ & $<0.001$ & 0.435 \\
\hline Duration to $3 \vee 6$ years & $1.06(1.05$ to 1.08$)$ & $<0.001$ & 0.036 \\
\hline Year first complete cycle started to 2006 v 2001* & $1.29(1.26$ to 1.32$)$ & $<0.001$ & 0.018 \\
\hline Tubal infertility to yes $v$ no & $0.80(0.78$ to 0.83$)$ & $<0.001$ & 0.007 \\
\hline Previous pregnancy in couple to no $v$ yes & 0.95 (0.93 to 0.98$)$ & $<0.001$ & 0.0001 \\
\hline \multicolumn{4}{|l|}{ Treatment information at first complete cycle } \\
\hline Cryopreservation of embryos to yes v no & $1.91(1.86$ to 1.96$)$ & $<0.001$ & 0.387 \\
\hline No of eggs collected to $13 v 5^{\star}$ & 1.29 (1.27 to 1.32$)$ & $<0.001$ & 0.378 \\
\hline Stage of embryos transferred: & & $<0.001$ & 0.123 \\
\hline Double cleavage & 1 & & \\
\hline No embryos transferred & $0.34(0.32$ to 0.36$)$ & $<0.001$ & \\
\hline Single cleavage & $0.57(0.54$ to 0.60$)$ & $<0.001$ & \\
\hline Single blastocyst & $1.07(0.93$ to 1.24$)$ & 0.35 & \\
\hline Double blastocyst & 1.79 (1.67 to 1.91$)$ & $<0.001$ & \\
\hline Triple cleavage & $1.02(0.98$ to 1.07$)$ & 0.36 & \\
\hline Triple blastocyst & $1.58(1.15$ to 2.14$)$ & 0.004 & \\
\hline Treatment type to ICSI $v$ IVF & 0.91 (0.89 to 0.93$)$ & $<0.001$ & 0.002 \\
\hline
\end{tabular}

ICSI=intracytoplasmic sperm injection.

*Interquartile odds ratio was calculated to aid interpretation of continuous predictors of age, duration of infertility, year first complete cycle started, and eggs collected. It is defined as the ratio of the odds of a live birth for the 75 th centile and the odds of a live birth for the 25 centile of the predictor.

Nagelkerke's $R^{2}$ (amount of explained variation in model) $=14.7 \%$; Tjur's coefficient of discrimination $=510.3 \%$. unexplained infertility or anovulation had slightly increased chances of success. Couples without a previous pregnancy had slightly decreased odds of live birth (0.93, 0.90 to 0.95). Couples who underwent ICSI had a $24 \%$ increased chance of a live birth than those who underwent IVF.

\section{Post-treatment model}

After the first fresh embryo transfer, the odds of a live birth increased with egg number, doubled in cases where embryos were cryopreserved, and decreased by $9 \%$ if the treatment was ICSI (table 3 ). The chances of a live birth over six complete cycles were lower after transfer of a single cleavage stage fresh embryo compared with transfer of a double cleavage stage fresh embryo. The effects of the predictors which also appeared in the pretreatment model were similar, apart from ICSI compared with IVF. Woman's age, cryopreservation of embryos, and number of eggs each explained a similar high amount of the total variation explained by all the factors (see supplementary table A3). Supplementary table A4 shows the results for women who had no eggs collected.

Assessing ability to predict cumulative live birth The $C$ index for the pretreatment model was 0.73 ( 0.72 to $0.74)$ and for the post-treatment model was 0.72 ( 0.71 to 0.73). The optimism adjusted calibration slope in both models was almost one (0.996 and 0.998, respectively) suggesting no overfitting of predictor effects.

\section{Examples of predicting cumulative live birth for specific couples}

Figure 3 shows an example of pretreatment predictions in different case scenarios. Part A shows the cumulative predicted probability of live birth over six complete cycles for couples starting ICSI for male factor infertility. The figure shows the probabilities for women aged 30 and 40 with either two or five years of infertility. While duration is less influential, younger women have a much higher chance of success, with a probability of 0.47 in complete cycle 1 (with two years' duration of infertility) increasing to 0.94 over six complete cycles ( 0.19 to 0.57 for 40 year old women). Similar predictions were found for couples with unexplained infertility who underwent IVF treatment in their first complete cycle.

Post-treatment, the predictions for the above scenarios were updated using information from the fresh treatment of the first complete cycle. The top line from figure 4 represents a woman with an excellent prognosis-aged 30 years, non-tubal infertility, 15 eggs collected, embryos cryopreserved, and a single fresh blastocyst transfer in the first complete cycle. The probability of success in complete cycle 1 is 0.68 . Cumulatively this increases to 0.89 over two complete cycles and rises to just under 1 over four complete cycles. The lower line in the chart represents a poorer prognosis-a woman aged 40 who had IVF, five eggs collected, no embryos frozen, and a single cleavage stage fresh embryo transferred in the first complete 

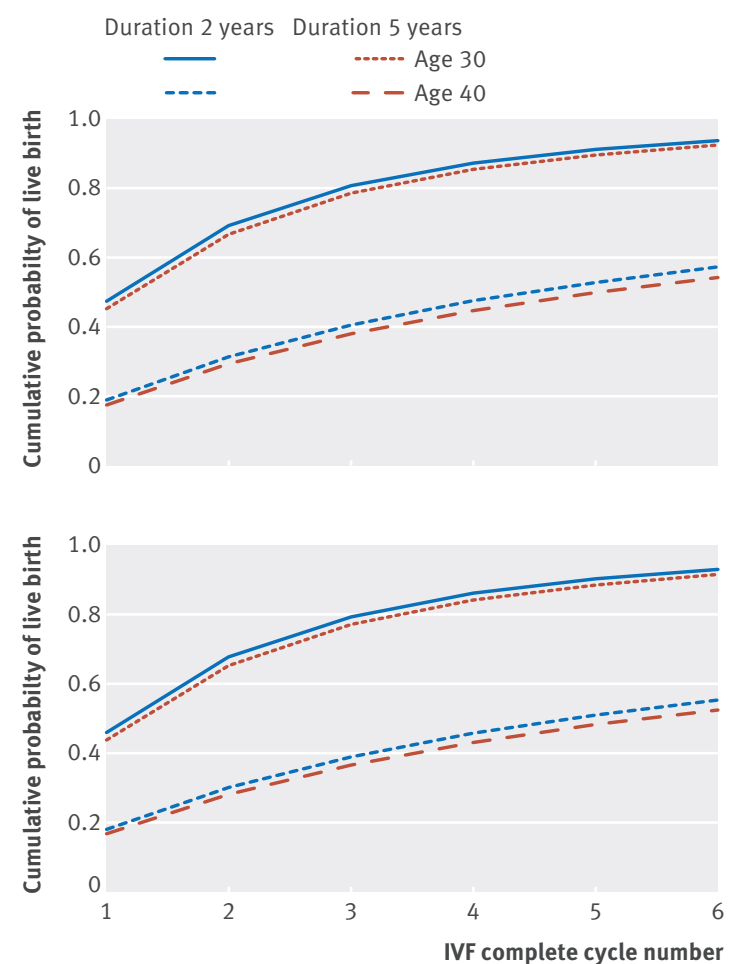

Fig 3 | Example of pretreatment model predicting cumulative probability of live birth over six complete cycles of in vitro fertilisation (IVF) for women aged $\mathbf{3 0}$ or $\mathbf{4 0}$ with two or five years of primary infertility who have: (A) male factor infertility and had treatment by intracytoplasmic sperm injection; (B) unexplained infertility and underwent IVF

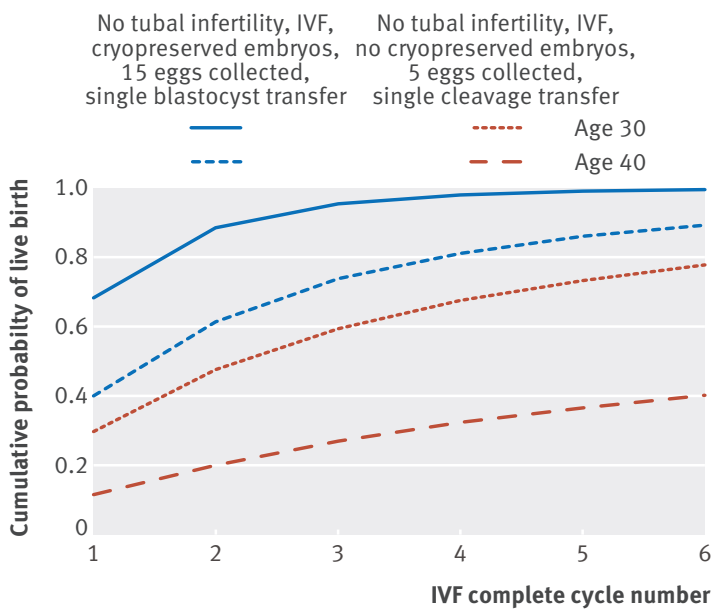

Fig 4 | Example of post-treatment model predicting cumulative probability of live birth over six complete cycles of in vitro fertilisation (IVF) for different patient and treatment characteristics (these patients have all had primary infertility for two years)

cycle. Her chance of a live birth is 0.11 in complete cycle 1 increasing to 0.20 over two complete cycles ( 0.41 over six). See supplementary text S3 for the formulas used to calculate the individualised cumulative predictions.

\section{Sensitivity analyses}

The effects from both models fitted using couples with complete data only were similar to those from the imputed models (see supplementary tables A5-A7).

In centres with low birth rates (2.5th centile of the random effect distribution), the odds of a live birth were 0.61 times that of the average, whereas in centres with high livebirth rates ( 97.5 th centile) the odds of a live birth were 1.65 times the average, after adjustment for the post-treatment predictors. The predictor effects from the models with the random intercept were similar to those from the fixed effects only model (see supplementary tables A8 and A9). The $\mathrm{C}$ indices were similar when fixed (from fixed effects model), marginal, or conditional (from random effects model) estimates of the predicted probabilities were used. A random slope for centre had no statistically significant effect.

\section{Discussion}

We have estimated the individualised chances of having a liveborn baby over a complete package of in vitro fertilisation (IVF) or intracytoplasmic sperm injection (ICSI) treatment at two time points. In couples embarking on IVF or ICSI we found that increasing age of the woman (from 30 years) was far the best predictor of live birth. After transfer of a fresh embryo in the first complete cycle, aside from the woman's age, increasing number of eggs collected and the cryopreservation of embryos were the next best predictors.

\section{Strengths and weaknesses of this study}

This study successfully predicted the chances of cumulative live birth over six complete cycles of IVF or ICSI using personalised information from national data over 10 years.

Treatment year was highly associated with live birth, signifying improvements in technology over time. Since the last year in our study period was 2008, we checked for underestimation of predictions owing to improvements in assisted conception since 2008. This was done by inflating the predictions from 2008 in the model to 2013, the latest year with published national livebirth rates on the Human Fertilisation and Embryology Authority website. ${ }^{24}$ The livebirth rate for each treatment cycle started using the woman's own fresh eggs was $25.8 \%$ in 2008 , and reduced to $25.2 \%$ in 2009 before slowly rising back to $25.8 \%$ in $2012 .{ }^{25}$ In 2013 the rate increased to $26.5 \%$. The intercept of the model was inflated by the difference between the 2008 and 2013 rates (on the log odds scale). Such an adjustment increased the predicted probabilities by up to a maximum of 0.009 (and by a mean of 0.007). In our new online calculator (https://w3.abdn.ac.uk/clsm/opis), predictions are computed using the 2013 estimate.

Unlike previous studies, we investigated heterogeneity in IVF centre effects using random effect intercepts as we had anonymised centre identifiers. Although there was some variation in the odds of live birth across centres, the ability of the model to predict using predictions conditioned on centres from the random effects model were no better than using standard estimates 
from the fixed effects model without adjustment for centre. Therefore, for reasons of generalisability, and because centre was anonymous, we advise using the fixed effects models.

Two models have been developed that may be used by clinicians at two different time points to estimate a couple's chances of having a live birth over one or more complete cycles of IVF. At these particular times points (before IVF and after first transfer of a fresh embryo) only information on the couple and treatment available at those times can be used to make predictions. Therefore at the point when the pre-IVF model will be used by clinicians to counsel couples as to their future chances of success, the woman's age, duration of infertility, type of infertility, previous pregnancy status of the couple, and treatment type are known. The post-IVF model revises these estimates using updated information from the first attempt at a fresh embryo transfer. We acknowledge that information from future complete cycles would provide more precise predictions, but our models reflect a more real life setting where such information is not available at the time of counselling. These models will provide an indication of the couple's future chances of achieving a live birth over a complete package of IVF at the beginning of treatment.

One limitation concerns the effect of calendar time on future frozen embryo transfers in the first complete cycle. For example, a woman may decide to have a frozen embryo transfer five years after the embryos were frozen. However, unless there were changes to her uterine environment, time is unlikely to decrease her chance of a live birth as the embryos were created using eggs retrieved five years earlier. Furthermore, we did not adjust for the time between complete cycles of IVF or ICSI nor for the change in predictors with each sequential complete cycle. The reason for this is because such information is not known at the time predictions are made.

We were unable to adjust for other potentially important predictors that were not available in the HFEA dataset. These include body mass index of the woman, ethnicity, smoking status, alcohol intake, and measures of ovarian reserve such as antral follicle count. A systematic review concluded that women's age, duration of infertility, number of eggs collected, and basal follicle stimulating hormone level were the most important predictors of IVF or ICSI success. ${ }^{12}$ Of these, only the last predictor was unavailable in our analysis. Smoking and alcohol consumption in both sexes have been shown to have an association with IVF success..$^{26-28}$ Although the availability of these potential predictors would have been valuable, the quality and completeness of routinely collected data on smoking and alcohol is questionable owing to being self reported. ${ }^{29} 30$

One of the model assumptions was that those couples who discontinue treatment without a live birth still have the same chance of a live birth as those who continue, after correction for predictor effects. This assumption only applies to women with obvious barriers to continuing treatment, such as a lack of funds, divorce, or death. However, most discontinue because they have a lower chance of success. ${ }^{31}$ The reason for treatment withdrawal was unfortunately not available. Studies have tried to discount optimal estimates by the same fixed percentage across all individuals. ${ }^{1132}$ However, such an approach is flawed because the chances of continuing treatment are highly likely to vary widely depending on a couple's characteristics (see supplementary text S2). By using a modelling approach to predict cumulative live birth we have adjusted for treatment continuation based on the covariates (such as the woman's age) as available.

Internal validation of the models showed that the optimism in the calibration slopes was minimal (owing to the large sample size). Because our model was developed using the UK national IVF registry database with the intention of being used in the UK there is no urgent need to externally validate on data from another geographical location, ${ }^{33}$ especially since IVF practice and policy varies across countries. Before the model is applied in other settings we recommend validation, specifically an assessment of the calibration of the model predictions. In the future, temporal validation on an up-to-date UK database is important to ensure that the model is robust to changes in UK IVF policy. Further work will involve sourcing a suitable database with which to carry out such a study.

\section{Strengths and weaknesses in relation to previous studies}

Many studies have reported the chance of a live birth after IVF or ICSI. ${ }^{1334-36}$ However, for different reasons they do not predict cumulative live birth over multiple complete cycles of IVF or ICSI. They either make predictions for the first transfer of a fresh embryo only, ${ }^{34}$ make predictions for individual embryo transfer episodes but with no linkage between cycle and woman (a necessary requirement for calculating cumulative outcomes over multiple cycles), ${ }^{13}$ or only adjust for the number of previous failed cycles. ${ }^{36}$ One study ${ }^{37}$ produced a similar model to ours, but this did not reflect usual practice as it only made cumulative predictions up to three fresh embryo transfer attempts and excluded any associated frozen embryo transfers. In an age where the emphasis is on reducing multiple births and increasing single embryo transfers, ${ }^{2}$ a complete cycle definition aids interpretability of results for clinicians and couples who want to know the chances of a live birth over an entire IVF programme. ${ }^{38}$

For fertility models, calibration in an external cohort tends to be the more important measure of performance than discrimination owing to the homogeneity of the population being studied. ${ }^{39}$ Discrimination assessments tend to result in $\mathrm{C}$ indices of around 0.65 at best. However, the $\mathrm{C}$ index for both models is, as far as we know, the highest found for IVF models to date. This might be due to the large sample size, carefully capturing non-linearity, the effect of complete cycle number, or a combination of the three.

\section{Interpretation and clinical importance}

These are the first models to predict individualised chances of live birth over a course of complete IVF cycles. 
The results are relevant not only for individual couples and their clinicians but also for funders and policy makers in determining access to state or insurance funded IVF. $^{2}$ The models have been converted into an online calculator that can be used to estimate the probability of live birth based on the characteristics of the couple and treatment (https://w3.abdn.ac.uk/clsm/opis).

The most appropriate use of these models is to serve as a counselling tool to help shape expectations for couples who would ideally like to know their overall chance of having a baby over a complete IVF or ICSI journey. As an example, the probability of a live birth in the first complete cycle for a couple with primary unexplained infertility for two years and the woman aged 30 undergoing IVF is 0.46 ( 0.93 over six complete cycles). After transfer of a single cleavage embryo following the collection of five eggs and no embryos cryopreserved, the probability decreases to 0.29 ( 0.78 over six complete cycles). Before treatment is started, clinicians would use these predictions to support their clinical knowledge when communicating to couples their chance of successful treatment over a complete IVF programme. Following the first embryo transfer, when treatment information is available, clinicians can then use the revised predictions to offer reassurance to couples about their future chances over multiple complete cycles. Existing models that only inform the chances of a liveborn baby after the first fresh cycle are not useful because the first treatment does not always succeed. The results from our model might help couples plan their time and prepare emotionally and financially for their complete IVF journey.

Currently we do not advise that these models be used to make decisions around whether or not couples should have IVF treatment. The reason for this is because their impact on clinical pathways, health outcomes, and costs need to be investigated and shown to be beneficial before doctors and couples can be persuaded to use them for decision making. ${ }^{4041}$ Furthermore, for a model to be truly useful for decisions around whether or not to treat it needs to provide estimates of the change in the absolute benefit of treatment over time, which would require information on couples under expectant management-that is, do not receive treatment. ${ }^{42}$ Unfortunately, such information is not available in the HFEA database or elsewhere at a national level.

We confirmed the reduction in the chance of live birth with increasing age, increasing duration of infertility, and previous pregnancy. ${ }^{13} 3536$ Before starting IVF or ICSI, unexplained infertility and anovulation were associated with an increased chance of live birth, whereas male factor and tubal infertility had a negative association. However, apart from tubal infertility these associations disappeared when predictions were updated using additional information from the first fresh embryo transfer. This suggests that treatment information overrides the effect of the different types of infertility-women with a high number of eggs who have cryopreserved embryos might reflect mild infertility encompassing most women in the unexplained, male factor, and anovulation categories.

\section{Further research}

In addition to temporal validation, external validation on national registry data from other countries might realise the potential of our models to be recalibrated (if required) and used in those populations.

\section{Conclusions}

Many couples with fertility problems embarking on IVF will undergo several complete cycles-each involving a fresh embryo transfer potentially followed by one or more frozen embryo transfers in order to achieve their first live birth. This study has developed a novel calculator that can predict the cumulative chances of a live birth for an individual couple over a package of complete IVF cycles. This tool will help to shape couples' expectations allowing them to plan their treatments more efficiently and to prepare emotionally and financially.

We thank the Human Fertilisation and Embryological Authority for permission to analyse their database, extracting the requested information, and assisting with our queries in an efficient manner; Nathan Staudt, Software Engineer at the University of Aberdeen, for developing the OPIS online calculator; and the data management support of the Grampian Data Safe Haven (DaSH) and the associated financial support of NHS Research Scotland, through NHS Grampian investment in the Grampian DaSH (www.abdn.ac.uk/iahs/facilities/ grampian-data-safe-haven.php).

Contributors: DJM, SB, AJL, EWS, and EtV designed the study. DJM conducted the statistical analysis and literature search and wrote the article. All authors contributed intellectually to the writing or revising of the manuscript, and approved the final version. DJM is the guarantor.

Funding: This work was supported by a Chief Scientist Office postdoctoral training fellowship in health services research and health of the public research (ref PDF/12/06). The views expressed here are those of the authors and not necessarily those of the Chief Scientist Office. The funder did not have any role in the study design; the collection, analysis, and interpretation of data; the writing of the report; nor the decision to submit the paper for publication. DJM had full access to all the data in the study and had final responsibility for the decision to submit for publication.

Competing interests: All authors have completed the ICMJE uniform disclosure form at www.icmje.org/coi_disclosure.pdf and declare: no support from any organisation for the submitted work; no financial relationships with any organisations that might have an interest in the submitted work in the previous three years; no other relationships or activities that could appear to have influenced the submitted work.

Ethical approval: Access to the anonymised Human Fertilisation and Embryological Authority database was approved by the north of Scotland research ethics committee (12/NS/0119).

Data sharing: No additional data available.

Transparency: The lead author (DJM) affirms that the manuscript is an honest, accurate, and transparent account of the study reported; that no important aspects of the study have been omitted; and that any discrepancies from the study as planned have been explained.

This is an Open Access article distributed in accordance with the Creative Commons Attribution Non Commercial (CC BY-NC 3.0) license, which permits others to distribute, remix, adapt, build upon this work non-commercially, and license their derivative works on different terms, provided the original work is properly cited and the use is non-commercial. See: http://creativecommons.org/licenses/ by-nc/3.0/

1 Oakley L, Doyle P, Maconochie N. Lifetime prevalence of infertility and infertility treatment in the UK: results from a population-based survey of reproduction. Hum Reprod 2008;23:447-50. doi:10.1093/ humrep/dem369. 
2 National Collaborating Centre for Women's and Children's Health. Fertility: assessment and treatment for people with fertility problems. 2013. https://www.nice.org.uk/guidance/cg156/evidence/ full-guideline-188539453.

3 Adamson GD, Tabangin M, Macaluso M, de Mouzon J. The number of babies born globally after treatment with the assisted reproductive technologies (ART). Fertil Steril 2013;100:S42. doi:10.1016/j. fertnstert.2013.07.1807.

4 Vrtacnik U, Vrtacnik Bokal E, Devjak R. Cumulative delivery rate after providing full reimbursement in vitro fertilization programme: a 6-years survey. Biomed Res Int 2014;2014:850478. doi:10.1155/2014/850478

5 Abuzeid MI, Bolonduro O, La Chance J, et al. Cumulative live birth rate and assisted reproduction: impact of female age and transfer day. Facts Views Vis Obgyn 2014;6:145-9.

6 Maheshwari A, McLernon D, Bhattacharya S. Cumulative live birth rate: time for a consensus?Hum Reprod 2015·30:2703-7.

$7 \quad$ Luke B, Brown MB, Wantman E, et al. Cumulative birth rates with linked assisted reproductive technology cycles. N Engl/ Med 2012;366:2483-91. doi:10.1056/NEJMoa1110238.

8 Macaldowie A, Wang YA, Chambers GM, Sullivan EA. National Perinatal Epidemiology and Statistics Unit, the University of New South Wales, Sydney. Assisted reproduction technology in Australia and New Zealand 2011. 2013. https://npesu.unsw.edu.au/surveillance/ assisted-reproductive-technology-australia-new-zealand-2011.

9 Malizia BA, Hacker MR, Penzias AS. Cumulative live-birth rates after in vitro fertilization. N Engl J Med 2009;360:236-43. doi:10.1056/ NEJMoa0803072

10 McLernon DJ, Maheshwari A, Lee AJ, Bhattacharya S. Cumulative live birth rates after one or more complete cycles of IVF: a populationbased study of linked cycle data from 178,898 women. Hum Reprod 2016;31:572-81. doi:10.1093/humrep/dev336.

11 Smith ADAC, Tilling K, Nelson SM, Lawlor DA. Live-birth rate associated with repeat in vitro fertilization treatment cycles. JAMA 2015;314:2654-62. doi:10.1001/jama.2015.17296.

12 van Loendersloot LL, van Wely M, Limpens J, Bossuyt PMM, Repping S, van der Veen F. Predictive factors in in vitro fertilization (IVF): a systematic review and meta-analysis. Hum Reprod Update 2010;16:577-89. doi:10.1093/humupd/dmq015.

13 Nelson SM, Lawlor DA. Predicting live birth, preterm delivery, and low birth weight in infants born from in vitro fertilisation: a prospective study of 144,018 treatment cycles. PLoS Med 2011;8:e1000386. doi:10.1371/journal.pmed.1000386.

14 Bhattacharya S, Maheshwari A, Mollison |. Factors associated with failed treatment: an analysis of 121,744 women embarking on their first IVF cycles. PLoS One 2013;8:e82249. doi:10.1371/journal. pone.0082249.

15 Human Fertilisation and Embryology Authority. Access anonymised HFEA data. 2013b. http://www.hfea.gov.uk/5874.html.

16 Sunkara SK, Rittenberg V, Raine-Fenning N, Bhattacharya S, Zamora J, Coomarasamy A. Association between the number of eggs and live birth in IVF treatment: an analysis of 400135 treatment cycles. Hum Reprod 2011;26:1768-74. doi:10.1093/humrep/der106.

17 Williams CL, Bunch KJ, Stiller CA, et al. Cancer risk among children born after assisted conception. N Engl J Med 2013;369:1819-27. doi:10.1056/NEJMoa1301675

18 Steyerberg EW. Clinical prediction models: a practical approach to development, validation and updating.Springer, 2009doi:10.1007/978-0-387-77244-8

19 te Velde ER, Nieboer D, Lintsen AM, et al. Comparison of two models predicting IVF success; the effect of time trends on model performance. Hum Reprod 2014:29:57-64. doi:10.1093/humrep/det393.

20 Bouwmeester W, Twisk JWR, Kappen TH, van Klei WA, Moons KGM, Vergouwe Y. Prediction models for clustered data: comparison of a random intercept and standard regression model. BMC Med Res Methodol 2013;13:19. doi:10.1186/1471-2288-13-19.

21 Harrell FE. Regression modeling strategies with applications to Linear Models, Logistic and Ordinal Regression, and Survival Analysis.Springer, 2001.

22 Thompson D. Ranking Predictors in logistic regression, SAS Institute: Paper D10-2009. 2009. http://www.mwsug.org/proceedings/2009/ stats/MWSUG-2009-D10.pdf.
23 Harrell FE Jr, , Lee KL, Mark DB. Multivariable prognostic models: issues in developing models, evaluating assumptions and adequacy, and measuring and reducing errors. StatMed 1996;15:361-87. doi:10.1002/ (SICI)1097-0258(19960229)15:4<361::AID-SIM168>3.0.CO;2-4.

24 Human Fertilisation and Embryology Authority. Fertility treatment in 2013: trends and figures. 2013a. http://www.hfea.gov.uk/docs/ HFEA_Fertility_Trends_and_Figures_2013.pdf.

25 Human Fertilisation and Embryology Authority. Facts and figures for researchers and the media. 2015. http://www.hfea.gov.uk/99.html.

26 Joesbury KA, Edirisinghe WR, Phillips MR, Yovich JL. Evidence that male smoking affects the likelihood of a pregnancy following IVF treatment: application of the modified cumulative embryo score. Hum Reprod 1998:13:1506-13. doi:10.1093/humrep/13.6.1506.

27 Soares SR, Melo MA. Cigarette smoking and reproductive function. Curr Opin Obstet Gynecol 2008;20:281-91. doi:10.1097/ GCO.0b013e3282fc9c1e

28 Rossi BV, Berry KF, Hornstein MD, Cramer DW, Ehrlich S, Missmer SA. Effect of alcohol consumption on in vitro fertilization. Obstet Gynecol 2011;117:136-42. doi:10.1097/AOG.0b013e31820090e1.

29 Boniface S, Kneale J, Shelton N. Drinking pattern is more strongly associated with under-reporting of alcohol consumption than socio-demographic factors: evidence from a mixed-methods study. BMC Public Health 2014;14:1297. doi:10.1186/1471-2458-14-1297.

30 Håkonsen LB, Ernst A, Ramlau-Hansen CH. Maternal cigarette smoking during pregnancy and reproductive health in children: a review of epidemiological studies. Asian J Androl 2014;16:39-49. doi:10.4103/1008-682X.122351.

31 Te Velde ER, Koudstaal J, Eimers JM. Assisted conception for infertility. BMJ 1992;305:1097-8. doi:10.1136/bmj.305.6861.1097-d.

32 Stolwijk AM, Wetzels AMM, Braat DDM. Cumulative probability of achieving an ongoing pregnancy after in-vitro fertilization and intracytoplasmic sperm injection according to a woman's age, subfertility diagnosis and primary or secondary subfertility. Hum Reprod 2000:15:203-9. doi:10.1093/humrep/15.1.203.

33 Collins GS, Reitsma JB, Altman DG, Moons KGM. Transparent reporting of a multivariable prediction model for individual prognosis or diagnosis (TRIPOD): the TRIPOD Statement. BMC Med 2015;13:1. doi:10.1186/s12916-014-0241-z.

34 Dhillon RK, McLernon DJ, Smith PP, et al. Predicting the chance of live birth for women undergoing IVF: a novel pretreatment counselling tool. Hum Reprod 2016;31:84-92. doi:10.1093/humrep/dev268.

35 Leushuis E, van der Steeg JW, Steures P, et al. Prediction models in reproductive medicine: a critical appraisal. Hum Reprod Update 2009:15:537-52. doi:10.1093/humupd/dmp013.

36 van Loendersloot LL, van Wely M, Repping S, Bossuyt PMM, van der Veen F. Individualized decision-making in IVF: calculating the chances of pregnancy. Hum Reprod 2013;28:2972-80. doi:10.1093/humrep/ $\operatorname{det} 315$

37 Luke B, Brown MB, Wantman E, et al. A prediction model for live birth and multiple births within the first three cycles of assisted reproductive technology. Fertil Steril 2014;102:744-52. doi:10.1016/j. fertnstert.2014.05.020

38 Stern JE, Hickman TN, Kinzer D, Penzias AS, Ball GD, Gibbons WE. Can the Society for Assisted Reproductive Technology Clinic Outcome Reporting System (SART CORS) be used to accurately report clinic total reproductive potential (TRP)?Fertil Steril 2012;97:886-9. doi:10.1016/j.fertnstert.2012.01.091.

39 Coppus SFPJ, van der Veen F, Opmeer BC, Mol BWJ, Bossuyt PMM. Evaluating prediction models in reproductive medicine. Hum Reprod 2009;24:1774-8. doi:10.1093/humrep/dep109.

40 Reilly BM, Evans AT. Translating clinical research into clinical practice: impact of using prediction rules to make decisions. Ann Intern Med 2006;144:201-9. doi:10.7326/0003-4819-144-3-200602070-00009.

41 Moons KGM, Kengne AP, Grobbee DE, et al. Risk prediction models: II. External validation, model updating, and impact assessment. Heart 2012;98:691-8. doi:10.1136/heartjnl-2011-301247.

42 McLernon DJ, te Velde ER, Steyerberg EW, Mol BWJ, Bhattacharya S. Clinical prediction models to inform individualized decision-making in subfertile couples: a stratified medicine approach. Hum Reprod 2014;29:1851-8. doi:10.1093/humrep/deu173.

Supplementary information: Additional tables 